\title{
Meditation-induced modulation of brain response to transcranial magnetic stimulation
}

\section{Dear editors,}

Meditation can be conceptualized as a family of complex emotional and attentional regulatory training regimes developed for various ends, including the cultivation of well-being and emotional balance. Previous studies have demonstrated that meditation may have positive effects on self-awareness, emotion regulation, attention processes, and also structural neuroplasticity [1]. However, the underlying neurophysiology remains poorly understood. Transcranial magnetic stimulation combined with electroencephalography (TMS-EEG) is a non-invasive technique to probe brain activity and connectivity. We aim at establishing whether TMS-EEG can detect changes in brain reactivity during meditation practice and at evaluating whether these changes are specific to the achieved state and to stimulated cortical areas.

Our subject (M.R.) was a left-handed, 69 years old expert meditator with more than sixty thousand hours of meditation practice in the Tibetan Buddhist Nyingmapa tradition. We obtained his informed consent and the study was approved by the Ethics Committee of the University and University Hospital of Liege in Belgium. Three voluntary induced mental states were investigated in the following order respectively to an initial baseline resting condition: open presence meditation (objectless meditative state inducing an attenuated duality of object and subject), self-induced cognitive opacity (SICO - thought-less state, with higher cognitive functions reduced to a minimum), and a closed-eye drowsy state, in which the subject attained light sleep (stage N1).

TMS-EEG was performed as in our previous studies (e.g. [2]), using neuronavigation (Nexstim Plc, Finland) and a compatible 60-channels EEG amplifier (Eximia, Nexstim Plc, Finland). Stimulations were targeted to the medial parts of the left superior parietal and frontal (premotor area) gyri, as identified on the subject's T1 3D magnetic resonance imaging (MRI) and at least 230 stimulation pulses were delivered at a frequency randomly jittered between 0.4-0.5 Hz. TMS-evoked potentials (TEPs) were obtained by averaging a minimum of 130 artifact-free single trials for each session. To assess the effects of self-induced mental states on cortical reactivity, we employed three indices: (i) the Divergence Index [2], which computes on the broad band $(0.1$ and $45 \mathrm{~Hz})$ TEP the percentage of samples that differ significantly (data-driven non-parametric statistics) across all channels and latencies, and compares them with respect to normative test-retest variability previously reported in [2]; (ii) the power within the natural frequency [3] range of each stimulated target, which provides a circuit-specific index of cortical reactivity in awake healthy subjects; and (iii) the phaselocking factor computed on TEPs filtered between 8 and $40 \mathrm{~Hz}$, which provides an index of connectivity that has been shown to be very sensitive to shifts in the state of vigilance across the sleep-wake cycle [4].

The DI computed between each voluntarily induced mental state and the resting state was always higher than the empirical cut-off (1.67\%) established in a previous study [2], indicating that the observed changes were significantly larger than the physiological variability of TEPs (Fig. 1A). This result is noteworthy because, stimulation parameters being kept equal by means of MRI-guided neuronavigation, TEP typically show changes in their wave shape only in the case of strong manipulation of cortical excitability, such as the ones induced by pharmacological modulation [5] or sleep-wake transitions [6]. Since the DI only provides a crude assessment of overall changes in the brain response, we next examined changes in the natural frequencies 
specific for the two stimulated targets. The natural frequency reflects the tendency of different cortical areas to oscillate around a preferential rate upon receiving a perturbation, which provides a circuit-specific index of cortical reactivity in healthy awake subjects (e.g. [5]). As in previous studies, in the present experiments TMS evoked prominent slow $\beta$-band oscillations after stimulation of the parietal cortex and prominent fast $\beta / \gamma$-band oscillations after stimulation of the premotor cortex. During open presence meditation, we observed an enhancement with respect to baseline of the power of site- specific evoked oscillations, for parietal $(15-25 \mathrm{~Hz})$ and premotor $(25-35 \mathrm{~Hz})$ stimulation (Fig. 1B). This finding is consistent with the enhanced amplitude of high fast-frequency oscillations $(20-45 \mathrm{~Hz})$ evoked by auditory stimuli found with the same meditator during a nondual meditation ([7] Suppl. Fig. 5). In this earlier report the amplitude of evoked oscillation was also associated to the amplitude of large gamma-band activity during meditation. Consistently with previous studies [6], evoked oscillations were dampened during the drowsy state in both areas, when the subject attained sleep stage N1. Notably, during SICO the power of the natural frequencies showed a marked area-specific dissociation: the values remained above baseline during parietal stimulation, whereas we observed a marked suppression during premotor stimulation, falling well below drowsiness level (Fig. 1B). These selfinduced changes in target-specific reactivity were paralleled by analogous changes in overall connectivity as measured by the global phase locking-factor (PLF) computed across all scalp sites (Fig. 1C); the PLF increased during open presence and decreased during drowsiness in both areas but showed a prominent reduction only in the case of premotor stimulation during SICO. In previous studies, the PLF decreased with the level of consciousness across the wake-sleep cycle [4] and it increased within NREM sleep when a dream was reported, compared to when dream was not reported [8]. PLF increase during open presence meditation may reflect the state of particularly vivid awareness and monitoring of internai states as reported by the highly trained meditator, whereas its selective reduction in premotor cortex during SICO might be related to a weaker involvement of frontal areas during a state in which cognitive and thought-like activity is reduced to a minimum [9]. The present results, although limited to a single highly trained practitioner, show for the first time that local and global aspects of cortical reactivity, synchrony and phase locking can be wilfully modulated. Further neurophysiological and neuroimaging studies, such as the ones focused on default mode network activity [10] on larger samples would be useful to better understand the neural basis of these specific meditation states. However, these findings are relevant because they extend the use of TMS-EEG to the study of changes of the content of consciousness within the range of eye-opened wakefulness and because they corroborate and complement, by a causal and perturbational perspective, previous results obtained by means of spontaneous EEG recordings during meditation practice [7].

Panel A. The upper plot shows the Divergence Index values computed over the post-stimulus period (250 $\mathrm{ms}$ ) across three different, voluntarily induced, active/meditative states (open presence, SICO and drowsiness) and baseline resting state condition for parietal (red dots) and premotor (green dots) stimulation. The dashed line indicates the empirical cut-off (1.67\%) established in [2].

Middle and bottom plots show TMS-evoked potentials recorded at $\mathrm{FCz}$ electrode and $\mathrm{P} 1$ electrode in baseline condition (black traces) and during open presence, SICO and drowsiness, while targeting the premotor cortex (red traces) and the parietal cortex (green traces). For each plot, time-points where the two traces significantly differ $(\mathrm{p}<0.05)$ are underlined by horizontal black lines. Vertical dashed lines mark the time of TMS occurrence. 
A
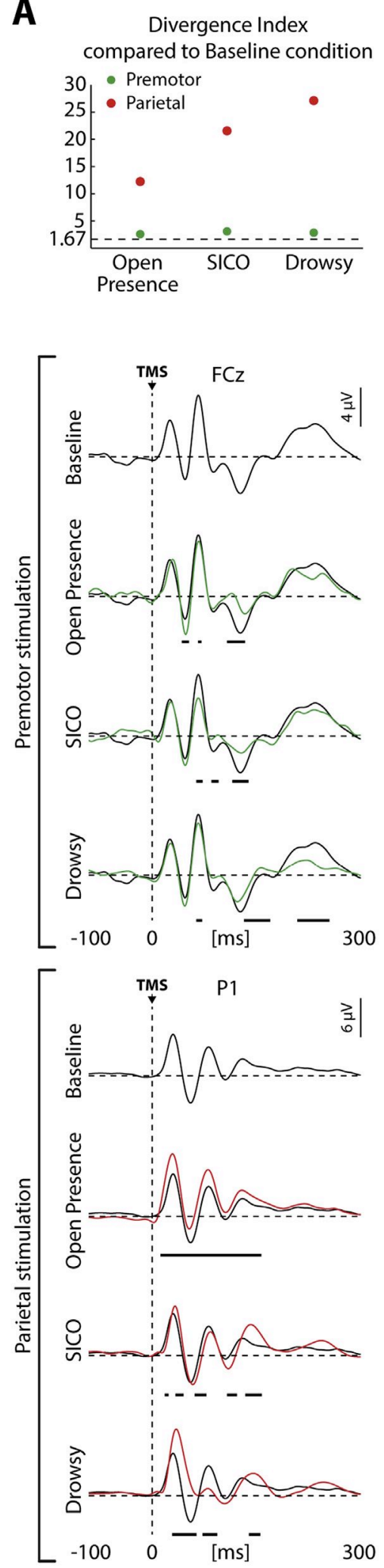

B
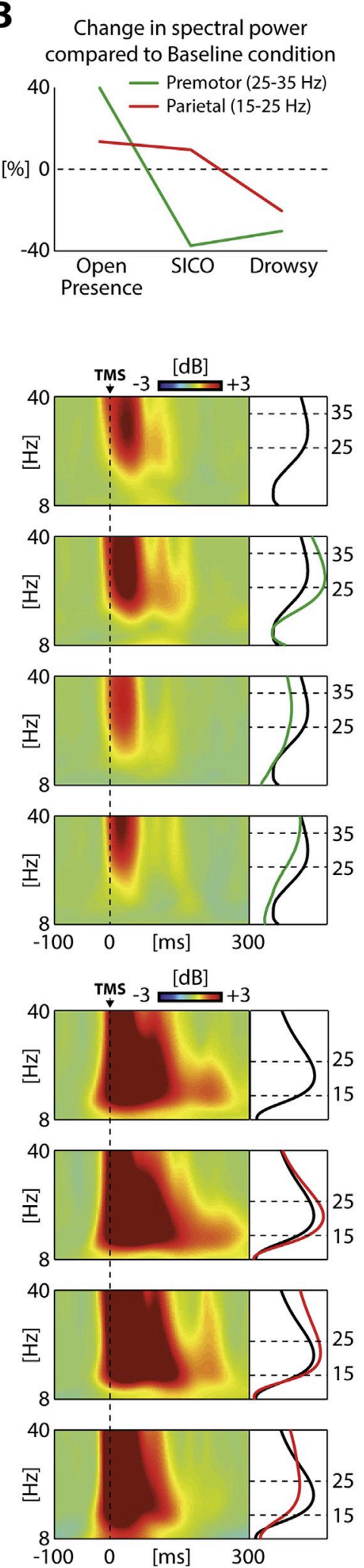

C

Change in PLF compared to Baseline condition

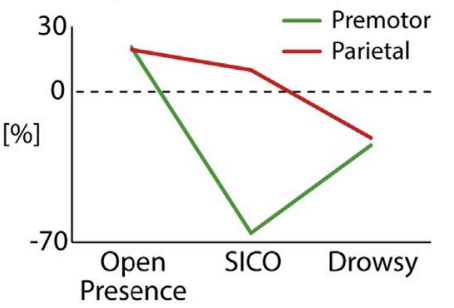

$$
\begin{aligned}
& \text { Easeline Condition } \\
& \text { Ective States }
\end{aligned}
$$
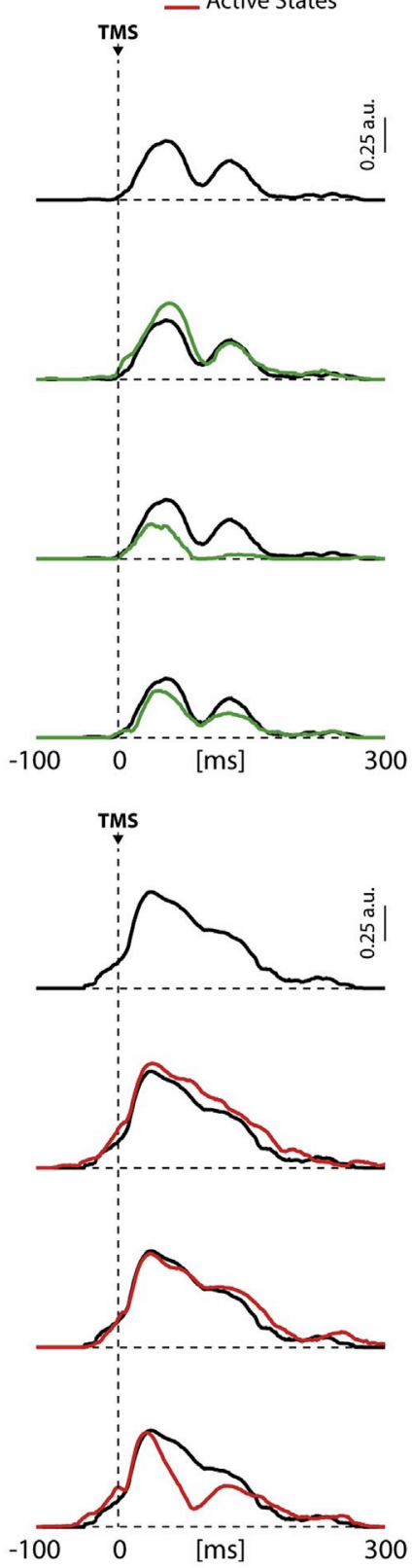

Fig. 1. TMS-EEG reveals modulation of brain activity under different voluntary induced states.

Panel B. Changes of spectral power across the three active/ meditative conditions (color-coded as in panel 
A) are expressed as percent difference compared to the baseline condition. Time- frequency spectra averaged over all channels and the corresponding power spectrum profile between 8 and $40 \mathrm{~Hz}$ evoked during the first $200 \mathrm{~ms}$ after TMS are shown together (dashed lines indicate the frequency band considered for comparison across conditions). Specifically, for each channel significant activation respectively to pre-stimulus activity (from -400 to $-100 \mathrm{~ms}$ ) is calculated by means of bootstrap statistics $(\alpha<0.05)$ and is colored in red, while the absence of any significant activation is colored in green.

Panel C. In the upper plot, changes of PLF across the three active/ meditative conditions (color-coded as in panel A) are expressed as percent difference compared to the baseline condition. For every condition and targeted cortical area (colored profiles) the PLF averaged over all channels are superimposed to PLF profiles calculated for baseline condition. By applying a statistical analysis assuming a Rayleigh distribution of the prestimulus values (from $-400 \mathrm{~ms}$ to $-100 \mathrm{~ms}$ ), PLF time points of each channel that were not significantly different $(\alpha<0.05)$ from pre-stimulus activity were set to zero.

\section{Funding}

This research was supported by the Belgian National Fund for Scientific Research (FNRS). This research has received funding from the European Union's Horizon 2020 framework program for Research and Innovation under the specific grant agreements No 785907 (Human Brain Project SGA2) and No 686764 (FETOPEN 2014-2015-RIA "Luminous"), Center-TBI, the James S. McDonnel Foundation Scholar Award 2013, European Space Agency, Belspo, B1AL Foundation, the Mind Science Foundation, the French Speaking Community Concerted Research Action (ARC-06/11340), and the University and University Hospital of Liege. OG is a post-doctoral researcher and $\mathrm{SL}$ a research director at the FRS- FNRS. The authors have no conflict of interest to declare.

\section{References}

[1] Tang Y-Y, Hölzel BK, Posner MI. The neuroscience of mindfulness meditation. Nat Rev Neurosci 2015;16:1-13. https://doi.org/10.1038/nrn3916.

[2] Casarotto S, Romero Lauro LJ, Bellina V, Casali AG, Rosanova M, Pigorini A, et al. EEG responses to TMS are sensitive to changes in the perturbation parameters and repeatable over time. PLoS One 2010;5, e10281. https:// doi.org/10.1371/journal.pone.0010281.

[3] Rosanova M, Casali AG, Bellina V, Resta F, Mariotti M, Massimini M. Natural frequencies of human corticothalamic circuits. J Neurosci 2009;29:7679-85. https://doi.org/10.1523/JNEUROSC1.0445-09.2009.

[4] Pigorini A, Sarasso S, Proserpio P, Szymanski C, Arnulfo G, Casarotto S, et al. Bistability breaks-off deterministic responses to intracortical stimulation during non-REM sleep. Neuroimage 2015;112:105-13. https://doi.org/10.1016/ j.neuroimage.2015.02.056.

[5] Premoli 1, Biondi A, Carlesso S, Rivolta D, Richardson MP. Lamotrigine and lev- etiracetam exert a similar modulation of TMS-evoked EEG potentials. Epilepsy Behav 2017;58:42-50. https://doi.org/10.1111/epi.13599.

[6] Massimini M, Ferrarelli F, Huber R, Esser SK, Singh H, Tononi G. Breakdown of cortical effective connectivity during sleep. Science 2005;309(80):2228-32. https://doi.org/10.1126/science.1117256.

[7] Lutz A, Greischar LL, Rawlings NB, Ricard M, Davidson RJ. Long-term meditators self-induce high-amplitude gamma synchrony during mental practice. Proc Natl Acad Sci U S A 2004;101:16369-73. https://doi.org/10.1073/ pnas.0407401101.

[8] Nieminen JO, Gosseries O, Massimini M, Saad E, Sheldon AD, Boly M, et al. Consciousness and cortical responsiveness : a within-state study during non-rapid eye movement sleep. Sci Rep 2016;6, 30932. https://doi.org/ 10.1038/srep30932.

[9] Perogamvros L, Baird B, Seibold M, Riedner B a, Boly M, Tononi G. The phenomenal contents and neural correlates of spontaneous thoughts across wakefulness, NREM sleep, and REM sleep. J Cognit Neurosci 2017;29: 1766-77. https://doi.org/10.1162/jocn_a_01155.

[10] Fingelkurts AA, Fingelkurts AA, Kallio-Tamminen T. Long-term meditation training induced changes in the 
operational synchrony of default mode network modules during a resting state. Cognit Process 2016;17:27-37. https://doi.org/10.1007/s10339-015-0743-4.

Olivier Bodart ${ }^{1}$

Giga-Consciousness, Coma Science Group, and Neurology Department, University and University Hospital of Liege, Belgium, Avenue de l'Hôpital 1, Liège, Belgium

Matteo Fecchio ${ }^{1}$

Department of Biomedical and Clinical Sciences "Luigi Sacco", University of Milan, Italy E-mail address: matteo.fecchio@unimi.it.

Marcello Massimini

Department of Biomedical and Clinical Sciences "Luigi Sacco", University of Milan, Italy

IRCCS Fondazione Don Gnocchi, Milan, ItalyE-mail address: marcello.massimini@unimi.it.

Sarah Wannez

Giga-Consciousness, Coma Science Group, and Neurology Department, University and University Hospital of Liege, Belgium, Avenue de l'Hôpital 1, Liège, Belgium E-mail address: sarah.wannez@,gmail.com.

Alessandra Virgillito

Giga-Consciousness, Coma Science Group, and Neurology Department, University and University Hospital of Liege, Belgium, Avenue de l'Hôpital 1, Liège, Belgium

Department of Translational Research on New Technologies in Medicine and Surgery, University of Pisa, Pisa, Italy E-mail address: alevirgi23@gmail.com.

Silvia Casarotto

Department of Biomedical and Clinical Sciences "Luigi Sacco",

University of Milan, Italy E-mail address: silvia.casarotto@unimi.it.

Mario Rosanova

Department of Biomédical and Clinical Sciences "Luigi Sacco",

University of Milan, Italy

Fondazione Europea di Ricerca Biomedica Onlus, Milan, ItalyE-mail address: mario.rosanova@unimi.it.

Antoine Lutz

Lyon Neuroscience Research Center, INSERM U1028, CNRS UMR5292,

Lyon 1 University, Lyon, France E-mail address: antoine.lutz@,inserm.fr.

Matthieu Ricard

Mind and Life Europe, Winterthur, Switzerland, \& Shechen Monastery,

Kathmandu, NepalE-mail address:mat108@gmail.com.

Steven Laureys

Giga-Consciousness, Coma Science Group, and Neurology Department, University and University Hospital of Liège, Belgium, Avenue de l'Hôpital 1, Liège,BelgiumE-mail address: steven.laureys@uliege.be.

Olivia Gosseries

Giga-Consciousness, Coma Science Group, and Neurology Department, University and University Hospital of Liège, Belgium, Avenue de l'Hôpital 1, Liège, Belgium E-mail address: ogosseries@uliege.be. 